\title{
Radiologische Verfahren bei gastrointestinaler Blutung - Diagnostik
}

\section{G. Layer}

\section{Radiological procedures in gastrointestinal bleeding - diagnostic}

Gastrointestinale Blutungen sind ein häufiges Problem der Notfalldiagnostik. Sie werden in 70-80\% durch Läsionen des oberen Gastrointestinaltraktes verursacht. Peptische Veränderungen, das MalloryWeiss-Syndrom und Ösophagusvarizen sind die häufigsten Blutungsquellen im oberen, Divertikel, Entzündungen, Angiodysplasien und Karzinome die häufigsten im unteren Gastrointestinaltrakt. Ihre Letalität wird mit 10-15\% bei großen Schwankungsbreiten abhängig von der Patientenauswahl angegeben (19). Patientenalter, Komorbidität, Blutverlust und Blutungsstadium nach Forrest bestimmen die Letalität $(9,5)$. Diagnostik und Therapie erster Wahl sind endoskopische Verfahren, mit denen die große Mehrzahl der GI-Blutungen nachgewiesen, lokalisiert und therapiert werden können.

Radiologische Verfahren sind nur in der Minderheit der Fälle indiziert, haben jedoch bei endoskopisch negativer Diagnostik und/ oder Therapie wachsende Bedeutung. Zur Verfügung stehen an erster Stelle interventionelle Kathetertechniken mit Hilfe der digitalen Subtraktionsangiographie (DSA). Daneben muss der Einsatz der digitalen Schnittbildtechniken Magnetresonanz- oder Kernspintomographie (MRT), Computertomographie (CT) und Sonographie einschließlich Farbdopplersonographie diskutiert werden. Konventionelle Röntgenverfahren wie Magen-Darm-Passage oder Enteroklysma haben dagegen nur geringe Aussagekraft (2). Zwar ist es mit der Dünndarm-Doppelkontrastdarstellung möglich, insbesondere distale blutende Dünndarmtumoren nachzuweisen, diese sind jedoch sehr selten ( $<1 \%$ aller Magen-Darm Tumoren). Die viel häufigeren Blutungsursachen bei mukosalen Läsionen und insbesondere Gefäßpathologien bleiben dem Verfahren verborgen.

\section{Schwer diagnostizierbare Blutungsquellen}

Der Haemosuccus pancreaticus als Komplikation einer meist chronischen Pankreatitis ist sehr selten. Es existieren zahlreiche Fallberichte dieses Krankheitsbildes. Wie häufig die transpapilläre Blutung insgesamt für eine gastrointestinale Blutung verantwortlich ist, bleibt aufgrund der geringen Inzidenz und fehlender Studien an ausreichend großen Patientenzahlen unklar. Suther

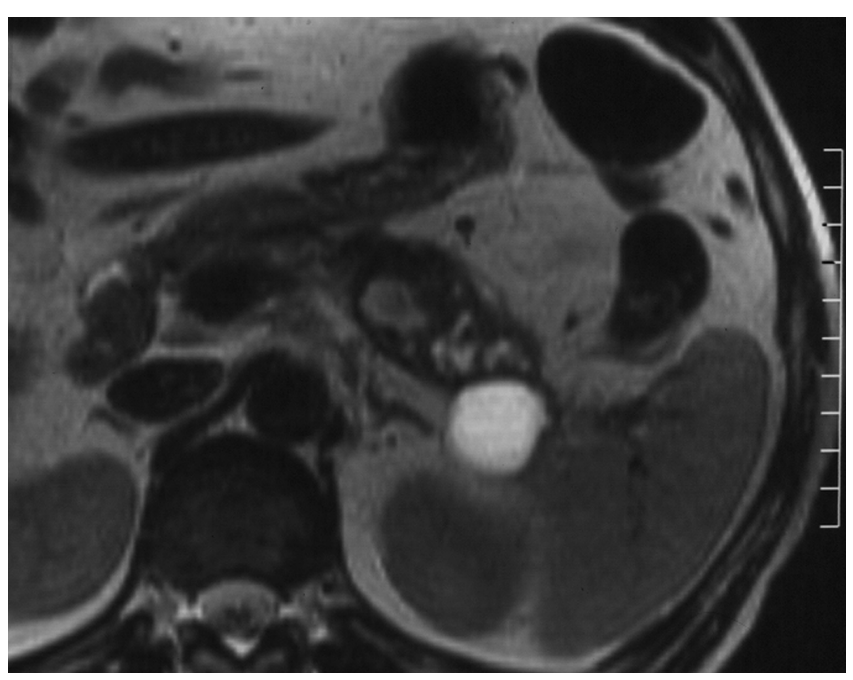

Abb. 1 MRT-Diagnostik bei dem im konkreten Fall geschilderten Patienten mit Haemosuccus pancreaticus (T2-gewichtete MRT-Dünnschichtaufnahme in transversaler Datenakquisition auf Höhe des Pankreasschwanzes). Das Organ wird signalarm (dunkel), Flüssigkeit signalreich (hell) abgebildet. Unmittelbar an die erweiterten unregelmäßigen Gangstrukturen im Pankreasschwanzbereich grenzt eine rundliche zystische Raumforderung. Der Befund ist typisch für eine Pankreasschwanz-Pseudozyste mit Einblutung bei chronischer Pankreatitis.

schätzte 1995 (18), dass man von einer Inzidenz von 0,5 pro 1000 Fälle einer akuten gastrointestinalen Blutung ausgehen muss.

Ein Haemosuccus tritt meist als Komplikation einer seit langem bekannten chronischen Pankreatitis auf. In über einem Drittel der Fälle kommt es zu Blutungen aus entzündlichen Pseudoaneurysmen der A. lienalis, aber auch der A. gastroduodenalis, der A. hepatica oder der A. pancreaticoduodenalis. Pathophysiologisch muss von einer enzymatisch entstehenden intrapankreatischen Gewebeläsion mit Arrosion intra- und/oder extrapankreatischer Gefäße mit Anschluss an den Pankreasgang ausge-

Institut

Zentralinstitut für Diagnostische und Interventionelle Radiologie (Direktor: PD Dr. G. Layer),

Klinikum Ludwigshafen gGmbH

Korrespondenz

Priv.-Doz. Dr. med. G. Layer · Direktor des Zentralinstituts für Diagnostische

und Interventionelle Radiologie

Klinikum Ludwigshafen gGmbH · Bremserstraße 79 • 67063 Ludwigshafen · Tel.: 0621/5034500

· Fax: 0621/5034537 ·.E-Mail: zir@klilu.de

eingereicht: 15.5.2003 - alkzeptiert: 21.8.2003

Bibliografie

Dtsch Med Wochenschr 2003;128:2074-2077 • c G Georg Thieme Verlag Stuttgart • New York • ISSN 0012-0472 


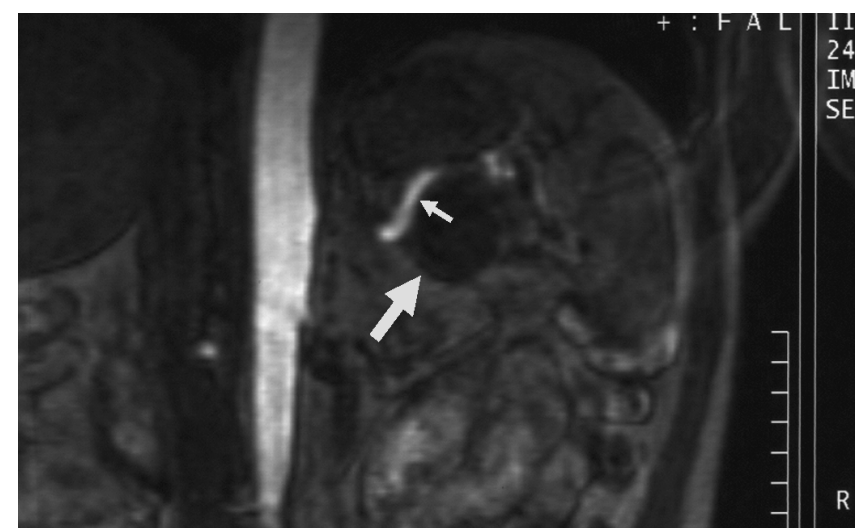

Abb. 2 MRT-Diagnostik bei dem im konkreten Fall geschilderten Patienten mit Haemosuccus pancreaticus (koronare Einzelschicht aus dem 3DDatensatz der kontrastgestützten MR-Angiographie des Abdomens). In dem MR-Angiographie-Datensatz werden alle Strukturen, in denen ein Blutfluss vorliegt, signalintensiv (hell) abgebildet. Die eingeblutete Zyste (großer Pfeil) bleibt dagegen als stationäres Gewebe signalarm wie die Umgebung. Man erkennt auf dem vorliegenden Einzelbild den unmittelbaren Bezug der schemenhaft abgrenzbaren Pankreasschwanz-Pseudozyste zum gebogenen Verlauf der Arteria lienalis (kleiner Pfeil).

gangen werden (Abb.1, Abb.2, Abb.3, Abb.4). Pseudoaneurysmen der Viszeralarterien sind bei Patienten mit chronischer Pankreatitis mit einer Häufigkeit von 10\% deutlich erhöht. Weitere mögliche Ursachen eines Haemosuccus pancreaticus sind maligne Tumoren und Pankreolithiasis. Unerkannt und unbehandelt hat das Krankheitsbild eine extrem schlechte Prognose mit einer Mortalität von bis zu 90\% (17).

Eine weitere seltene, aber mit extrem schlechter Prognose verbundene Blutungsursache sind aortointestinale Fisteln. Ihnen gehen in der Mehrzahl der Fälle Operationen an Aortenaneurysmen voraus, in den übrigen Fällen liegen inflammatorische Aneurysmen oder ein M. Behçet zugrunde.

Als dritte Gruppe schwer diagnostizierbarer gastrointestinaler Blutungen sind Arrosionsblutungen aus viszeralen Aneurysmen anzusprechen. Das am häufigsten betroffene Gefäß ist die A. lienalis (ca. 60\% aller Fälle), das höchste Rupturrisiko weist die A. gastrica sinistra (ca. 90\%) auf.

kurzgefasst: Schwer diagnostizierbare Ursachen gastrointestinaler Blutungen haben auch eine entsprechend schlechte Prognose. Zu ihnen sind insbesondere die transpapilläre Blutung bei Haemosuccus pancreaticus, die meist duodenale Blutung bei aortoenteraler Fistel und die Arrosionsblutung bei viszeralen Aneurysmen zu rechnen.

\section{Radiologische Lokalisationsverfahren}

\section{Angiographie}

Häufig wird die Diagnostik der okkulten d.h. der endoskopisch nicht sicher diagnostizierten und lokalisierten gastrointestinalen Blutung, wie dies auch bei unserem Patienten mit Haemosuccus pancreaticus der Fall war, durch eine Vielzahl von Nebenbefunden erschwert. So können Ösophagusvarizen, Zeichen einer Refluxösophagitis oder der Sigmadivertikulose die wahre Blutungsursache verschleiern.
$\mathrm{Zu}$ fordern ist von einem wertvollen diagnostischen Verfahren für die okkulte gastrointestinale Blutung der Nachweis, aber auch die exakte Lokalisation der Blutung. Ideal ist es, wenn das Verfahren dann auch in einer Sitzung therapeutisch genutzt werden kann. Diese Forderungen kann von allen radiologischen Verfahren nur die Angiographie erfüllen (Abb.3, Abb.4, Abb.5, Abb.6). Dabei ist bei der Bewertung der Ergebnisse des Verfahrens zu berücksichtigen, dass in der Regel eine Negativauswahl erfolgt ist, da endoskopische Verfahren erfolglos waren. Die in der Literatur angegebenen diagnostischen Sensitivitäten im Blutungsnachweis schwanken bei Studien mit größeren Fallzahlen zwischen 24 und 78\% $(1,2,10,11)$. Akute Blutungen werden häufiger angiographisch nachgewiesen als chronische oder chronisch rezidivierende (8). Für das blutungsfreie Intervall schwanken die angiographischen Nachweisraten zwischen $14 \%$ und $74 \%$, während bei manifester Hb-wirksamer Akutblutung 60-87\% erfolgreich radiologisch diagnostiziert werden konnten $(1,2,11)$. Bei chronisch rezidivierenden Blutungen ist der wiederholte Versuch des angiographischen Nachweises sinnvoll, allerdings dann möglichst auch in der akuten Blutungsphase (12).

Der Erfolg der DSA-Technik hängt selbstverständlich von einer adäquaten Technik ab. Hohe Bildfrequenz, Einsatz von Spasmolytika und, wenn erforderlich auch superselektive Katheterplatzierung müssen gefordert werden (Abb.5 und Abb.6). Die Blutungsprovokation durch selektive Applikation thrombolytischer oder vasodilatativer Pharmaka hat sich in der klinisch-radiologischen Routine nicht durchsetzen können, obwohl Erfolge berichtet wurden (13). Diese sind allerdings nicht unstrittig, wenngleich erstaunlicherweise die Provokation einer viszeralen Blutung durch vasoaktive Substanzen kein besonderes Risiko für den Patienten darzustellen scheint (3). Gleiches gilt für die $\mathrm{CO}_{2}$-DSA, bei der statt jodhaltigem Kontrastmittel reines $\mathrm{CO}_{2}$ Gas selektiv appliziert wird. Dieses hat aufgrund seiner $400 \times$ niedrigeren Viskosität und hohem Gewebekontrast eine gute Nachweisbarkeit des Kontrastmittelaustritts über die Gefäßwand und halbiert damit die für den Blutungsnachweis benötigte Blutverlustmenge (15). In DSA-Technik wird ansonsten davon ausgegangen, dass eine Blutung erst ab einer Blutungsmenge von ca. $0,5 \mathrm{ml} / \mathrm{min}$ nachgewiesen werden kann. Unterhalb dieser Blutungsaktivität können Gefäßabbrüche, Tumorgefäße oder der Nachweis früher venöser Abflüsse indirekte Hinweise auf Blutungen und Blutungslokalisation geben. Häufigste Blutungsquelle ohne direkten Extravasatnachweis sind Angiodysplasien gefolgt von Tumoren.

Wird eine Blutung angiographisch nachgewiesen, so ist aufgrund der anatomischen Zuordenbarkeit der Gefäße zu den Darmabschnitten praktisch immer eine exakte Lokalisation und damit ggf. eine Planung eines chirurgischen Eingriffs möglich. Das ist ein entscheidender Vorteil gegenüber nuklearmedizinischen Verfahren, für die zwar ähnliche Sensitivitäten im Blutungsnachweis angegeben werden, die aber eine anatomisch exakte Zuordnung nur in der Minderheit der Fälle ermöglichen (14).

Die klinische Relevanz einer angiographischen Blutungsdiagnostik wird dadurch dokumentiert, dass positiv angiographierte Patienten häufiger operiert werden, eine signifikant niedrigere Blutungspersistenz, Rezidivblutungsrate und geringere Mortalität haben im Vergleich zu negativ angiographierten Patienten (11). 


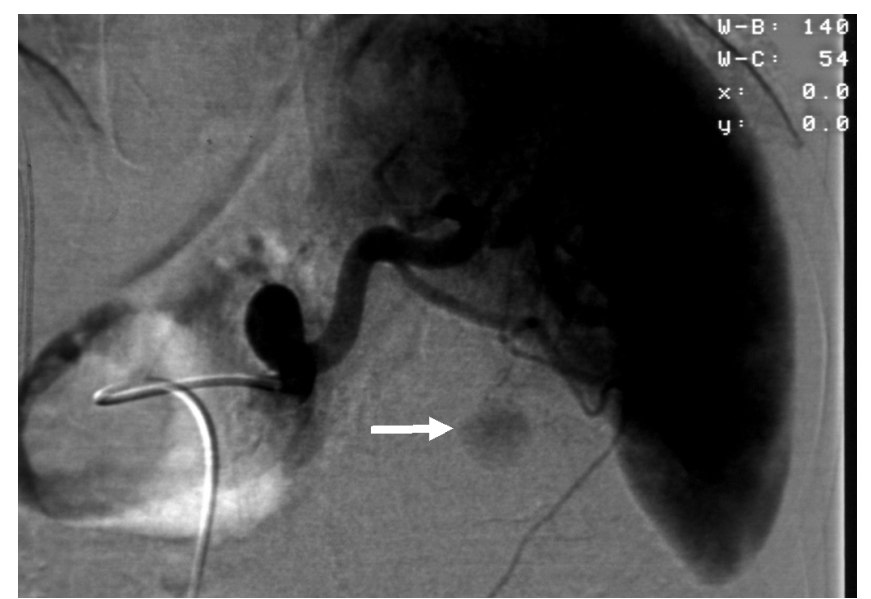

Abb.3 DSA-Diagnostik bei dem im konkreten Fall geschilderten Patienten mit Haemosuccus pancreaticus (Übersichtsangiographie der A. lienalis). Dargestellt sind die kräftigen Äste des Gefäßes im Bereich des Hilus der Milz und ihrer Aufzweigungen. Am Unterpol des Milzhilus sind schemenhaft feine Gefäße zu einer Rundstruktur (Pfeil) zu erkennen, die flau durch das KM angefärbt wird. Dieser Befund entspricht den arrodierten A.-lienalis-Gefäßen, die in die Pankreasschwanzpseudozyste einbluten.

\section{Computertomographie}

Die Computertomographie (CT) hat durch die Einführung immer schnellerer Geräte (Multislice-Technologie) mit der optimalen Erfassung einer maximalen Kontrastmittelanflutung im Mesenterium in letzter Zeit zunehmend Beachtung für den Blutungsnachweis bei okkulten gastrointestinalen Blutungen gefunden. Bei allerdings sehr kleinen Patientenzahlen konnte ein positiver korrekter Blutungsnachweis bei akuter unterer gastrointestinaler Blutung bei 15 von 19 bzw. 13 von 18 Patienten geführt werden $(6,7)$. Eine besondere Variante ist die selektive CT-Mesenterikographie, bei der der Patient mit in der A. mesenterica superior liegendem Katheter ins CT gebracht wird und dort nach einem selektiven Kontrastmittelbolus mit einer schnellen Bildfolge dünner Schichten untersucht wird. In einer kleinen Pilotstudie mit lediglich 8 Patienten konnte dabei die Überlegenheit dieser Technik gegenüber der konventionellen DSA gezeigt werden (16). Im Fall einer transpapillären Blutung wie bei Haemosuccus pancreaticus muss darauf geachtet werden, dass neben der A. mesenterica superior und ihren Ästen auch die lienalen Äste und Kommunikationen über den Truncus coeliacus mit dargestellt werden.

\section{Magnetresonanztomographie}

Ein zweites unerlässliches radiologisches Verfahren bei Patienten mit schwer diagnostizierbarer Blutungsquelle ist die Magnetresonanztomographie (MRT) einschließlich MR-Angiographie und MRCholangiopankreatikographie. Hiermit können Pseudozysten des Pankreas, Füllungsdefekte im Pankreasgangsystem, der Bezug von Zysten zum Pankreasgang und zu den Gefäßen sowie der Charakter der zystischen Läsion - insbesondere ob eingeblutet oder nicht - sicher charakterisiert werden. Die MRT hat in unserem Institut die vormals durchgeführte CT abgelöst, da bei der MRT in einem Untersuchungsgang sowohl eine effiziente Schnittbilddiagnostik mit näherer Charakterisierung des Zysteninhaltes, als auch eine Gefäßdiagnostik und eine Gallenwegsdiagnostik stattfinden kann.

Die besonderen Vorteile der MRT liegen in fehlender Strahlenexposition, beliebiger räumlicher Schnittführung, höchstem Weichteilkontrast und der Möglichkeit, in einer Sitzung diagnostische

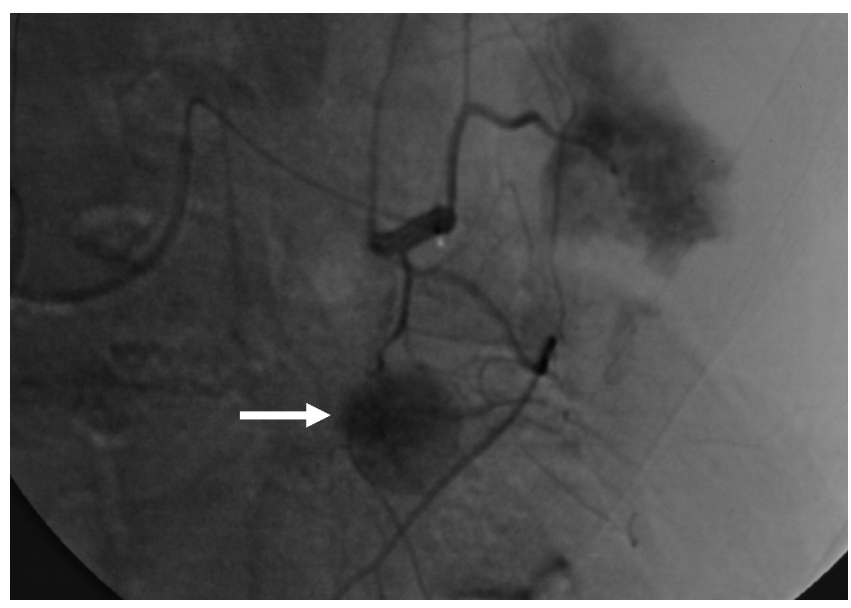

Abb.4 DSA-Diagnostik bei dem im „konkreten Fall“ geschilderten Patienten mit Haemosuccus pancreaticus. Superselektive Darstellung des in Abb. 1 bereits angiographierten Gefäßsystems der A. lienalis. Nach superselektiver Katheterisierung der arrodierten lienalen Gefäße ist die Einblutung in die Pankreasschwanz-Pseudozyste (Pfeil) definitiv nachweisbar.

Schnittbilder, aber auch MR-Angiographie- und MR-Cholangiopankreatikographie-Datensätze zu erhalten. Damit ist eine exakte Charakterisierung von Geweben und Flüssigkeiten mit guter räumlicher Zuordnungsmöglichkeit insbesondere in Bezug zum Gefäßsystem und Gallenwegs- und Pankreasgangssystem möglich.

\section{Einordnung radiologischer Verfahren}

Aus dem vorher Beschriebenen kann abgeleitet werden, dass die DSA die größte Bedeutung für den Nachweis und die Lokalisation im Rahmen der radiologischen Verfahren besitzt (Tab.1). Die Einordnung von Endoskopie, Szintigraphie und Kapselendoskopie erfolgt fachfremd subjektiv im Vergleich mit den radiologischen Verfahren ohne Anspruch auf objektive Beweisbarkeit. Bei der Einschätzung der Kapselendoskopie überwiegt sicherlich die Begeisterung für ein neues innovatives Verfahren, ohne dass evidenzbasierte Ergebnisse heute bereits in ausreichendem Maß vorliegen.

kurzgefasst: In der radiologischen Diagnostik der okkulten gastrointestinalen Blutung spielen drei Verfahren eine besondere Rolle: die selektive Katheterangiographie in DSA-Technik, Magnetresonanzverfahren einschließlich MR-Angiograpie und MRCP und MultisliceSpiral-CT möglichst als direkte CT-Mesenterikographie.

Tab. 1 Diagnostische Verfahren zum Nachweis unklarer gastrointestinaler Blutungen.

\begin{tabular}{|c|c|c|}
\hline Verfahren & $\begin{array}{l}\text { Diagnostische } \\
\text { Treffsicherheit }\end{array}$ & Blutungslokalisation \\
\hline Sonographie & $(-)$ & $(+)$ \\
\hline Röntgentechniken & & $(+)$ \\
\hline$C T$ & + & + \\
\hline MRT & + & ++ \\
\hline$D S A$ & ++ & +++ \\
\hline Szintigraphie & +++ & $(+)$ \\
\hline Endoskopie & ++ & +++ \\
\hline Kapselendoskopie & +++ & +++ \\
\hline
\end{tabular}



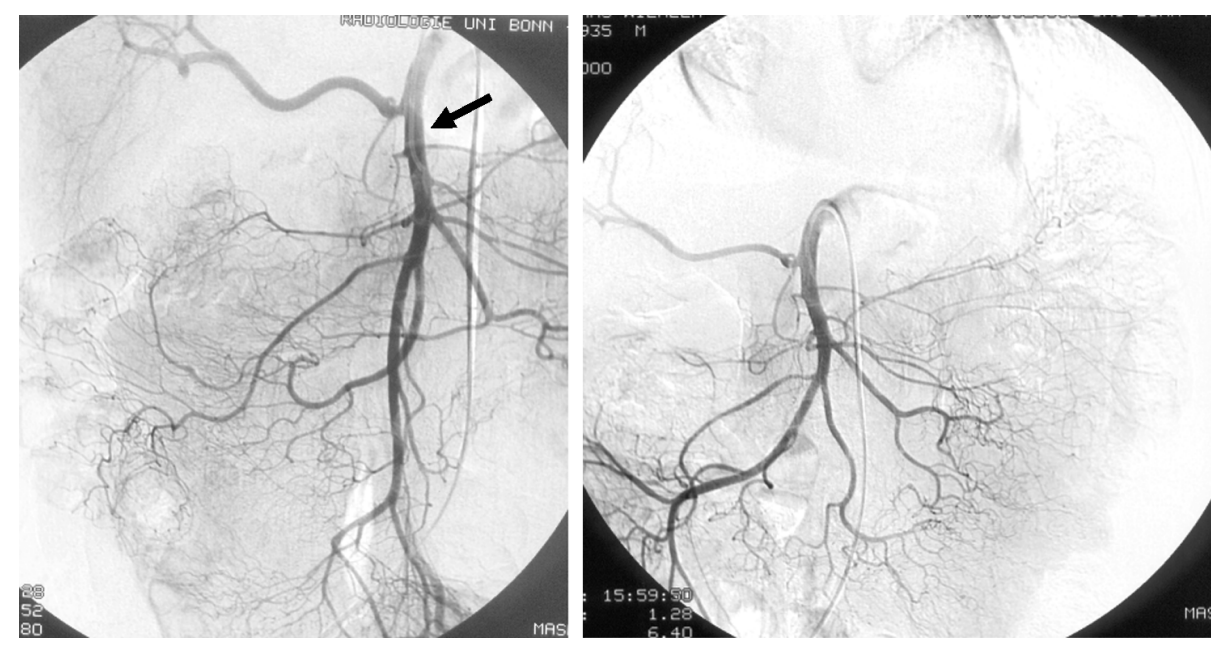

Abb.5 Unauffällige Übersichtsbilder einer Mesenterikographie bei gastrointestinaler Blutung. Der Katheter (Pfeil) liegt im Hauptstamm der A. mesenterica superior, die als Variante auch die rechte Leberarterie abgibt.
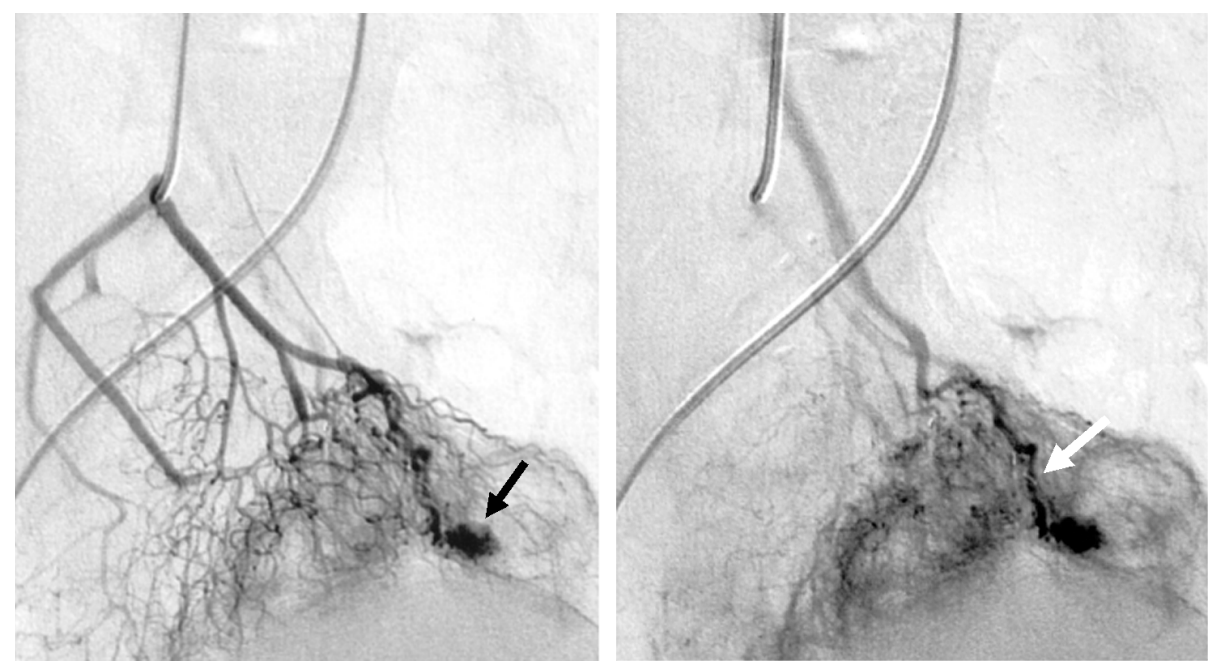

Abb. 6 Superselektive Mesenterikographie mit Koaxialkatheter bei gastrointestinaler Blutung (gleicher Patient wie Abb.5.) Der Koaxialkatheter wird bis unmittelbar an den Nidus einer Gefäßmissbildung (schwarzer Pfeil) vorgeführt. Links sind in der frühen noch arteriellen Phase die kräftigen Feederarterien und der Nidus der Missbildung zu erkennen, rechts in der frühen venösen Phase die typische sog. „frühe Vene" (weißer Pfeil) und der erweiterte venöse Abstrom.
Autorenerklärung: Der Autor erklärt, dass er keine finanziellen Verbindungen mit einer Firma hat, deren Produkt in dem Artikel eine wichtige Rolle spielt (oder mit einer Firma, die ein Konkurrenzprodukt vertreibt).

\section{Literatur}

1 Allison DJ, Hemingway AP, Cunningham DA. Angiography in gastrointestinal bleeding. Lancet 1982; 2: 30-33

2 Antes G, Neher M, Hiemeyer V, Burger A. Gastrointestinal bleeding of obscure origin: role of enteroclysis. Eur Radiol 1996; 6: 851-854

3 Bloomfeld RS, Smith TP, Schneider AM, Rockey DC. Provocative angiography in patients with gastrointestinal hemorrhage of obscure origin. Am J Gastroenterol 2000; 95: 2807-2812

4 Bonacker MJ, Begemann PGC, Dieckmann C, Yekebas E, Adam G. Stellenwert der Angiographie in der Diagnose und Therapie gastrointestinaler Blutungen. Fortschr Röntgenstr 2003; 175: 524-531

${ }^{5}$ Dertinger SH, Vestner H, Muller K, Merz M, Hahn EG, Altendorf-Hofmann A, Ell C. Prospektive Untersuchung zu Diagnostik, Therapie und Verlauf der akuten gastrointestinalen Blutung bei 397 Patienten. Wien Klin Wochenschr 1996; 108: 717-721

${ }^{6}$ Ernst O, Bulois P, Saint-Drenant $S$ et al. Helical CT in acute lower gastrointestinal bleeding. Eur Radiol 2003; 13: 114-117

7 Ettorre GC, Francioso G, Garribba AP et al. Helical CT angiography in gastrointestinal bleeding of obscure origin. Am J Roentgenol 1997; 168: 727-731

8 Fiorito JJ, Brandt LJ, Kozicky O et al. The diagnostic yield of superior mesenteric angiography: correlation with the pattern of gastrointestinal bleeding. Am J Gastroenterol 1989; 84: 878-881

9 Forrest JA, Finlayson ND, Shearman DJ. Endoscopy in gastrointestinal bleeding. Lancet 1974; 2: 394-397
10 Gunderman R, Leef J, Ong K, Reba R, Metz C. Scintigraphic screening prior to visceral arteriography in acute lower gastrointestinal bleeding. J Nucl Med 1998; 39: 1081-1083

11 Heider J, Layer G, Textor HJ, Schild HH. Beeinflusst die angiographische Blutungslokalisation den klinischen Verlauf und die Mortalität bei unklarer gastrointestinaler Blutung? Aktuel Radiol 1998; 8: 71-75

12 Lau WY, Ngan H, Chu KW, Yuen WK. Repeat selective visceral angiography in patients with gastrointestinal bleeding of obscure origin. $\mathrm{Br}$ J Surg 1989; 76: 226-229

13 Ryan JM, Key SM, Dumbleton SA et al. Nonlocalized lower gastrointestinal bleeding: provocative bleeding studies with intraarterial tPA, heparin, and tolazolin. J Vasc Interv Radiol 2001; 12: 1273-1277

14 Rollins ES, Picus D, Hicks ME et al. Angiography is useful in detecting the source of chronic gastrointestinal bleeding of obscure origin. AJR Am J Roentgenol 1991; 156: 385-388

15 Sandhu C, Buckenham TM, Belli AM. Using CO2-enhanced arteriography to investigate acute gastrointestinal hemorrhage. AJR AM J Roentgenol 1999; 173: 1399-1401

16 Schürmann K, Bücker A, Jansen M et al. Selektive CT-Mesentericographie zur Diagnostik schwerer Darmblutungen unklaren Ursprungs: Vorläufige Ergebnisse. Rofo Fortschr Geb Röntgenstr Neuen Bildgeb Verfahr 2002; 174: 444-451

17 Storr M, Weigert N, Weiß W et al. Haemosuccus pancreaticus - Eine seltene Komplikation der Pankreatitis. Dtsch Med Wochenschr 2001; 126: $108-112$

${ }^{18}$ Suter M, Doenz F, Chapuis G, Gillet M et al. Haemorrhage into the pancreatic duct (hemosuccus pancreaticus): recognition and management. Eur J Surg 1995; 161: 887

19 Wilcox CM, Clark WS. Causes and outcome of upper and lower gastrointestinal bleeding: the Grady Hospital experience. South Med J 1999; 92: 44-50 